\title{
DEEP CIRCULATION IN RED GIANT STARS: A SOLUTION TO THE CARBON AND OXYGEN ISOTOPE PUZZLES?
}

\author{
G. J. WASSERBURG \\ The Lunatic Asylum, Charles Arms Laboratory 170-25, California Institute of Technology, Pasadena, CA 91125 \\ ARNOLD I. BOOTHROYD \\ Canadian Institute for Theoretical Astrophysics, University of Toronto, \\ 60 St. George Street, Toronto, Ontario M5S 1A7, Canada \\ AND \\ I.-JULIANA SACKMANN \\ W. K. Kellogg Radiation Laboratory 106-38, California Institute of Technology, Pasadena, CA 91125 \\ Received 1995 March 6; accepted 1995 April 25
}

\begin{abstract}
The long-standing puzzle of low ${ }^{12} \mathrm{C} /{ }^{13} \mathrm{C}$ in low-mass red giant branch (RGB) stars, and the more recent puzzle of low ${ }^{18} \mathrm{O} /{ }^{16} \mathrm{O}$ ratios in asymptotic giant branch (AGB) stars and in circumstellar $\mathrm{Al}_{2} \mathrm{O}_{3}$ grains preserved in meteorites, can be resolved by deep circulation currents below the bottom of the standard convective envelope. These currents transport matter from the nonburning bottom of the convective envelope down to regions where some CNO processing can take place ("cool bottom processing"). Modeling circulation with separate downward and upward streams, we found that, to resolve both discrepancies, the base of the extra mixing had to reach a temperature $T_{P}$ close to that of the H-burning shell, namely, $\Delta \log T \approx 0.17$ from the base of the H-shell for both RGB and AGB stars. While the envelope composition depends sensitively on $T_{P}$, it is insensitive to the speed or geometry of mixing. This indicates that our stream circulation model is generic, so that more sophisticated mixing models with the same $T_{P}$ would yield similar results. On the AGB, our models predict that stars with $\operatorname{low}{ }^{18} \mathrm{O} /{ }^{16} \mathrm{O}$ can be either $\mathrm{S}$ or $\mathrm{C}$ stars but must have low ${ }^{12} \mathrm{C} /{ }^{13} \mathrm{C}(\sim 4)$ and elevated ${ }^{14} \mathrm{~N}$. Cool bottom processing also destroys ${ }^{3} \mathrm{He}$, so that galactic $\left(\mathrm{D}+{ }^{3} \mathrm{He}\right)$ decreases with time; this removes the strongest lower limit on the baryon density $\Omega_{b}$ from big bang nucleosynthesis models.
\end{abstract}

Subject headings: early universe - nuclear reactions, nucleosynthesis, abundances - stars: abundances — stars: late-type

\section{INTRODUCTION}

It is known that ${ }^{12} \mathrm{C} /{ }^{13} \mathrm{C}$ in low-mass red giants cannot be accounted for by standard stellar evolution theory (see Dearborn, Eggleton, \& Schramm 1976; Gilroy 1989). In "first dredge-up," the ${ }^{13} \mathrm{C}$-rich pocket from main-sequence burning is brought to the surface by the star's deep convective envelope; for low-mass stars, this occurs near the base of the red giant branch (RGB). While theoretical predictions of ${ }^{12} \mathrm{C} /{ }^{13} \mathrm{C}$ agree with observations within $\sim 25 \%$ for stellar masses $\gtrsim 2$ $M_{\odot}$, the predicted trend between 1 and $2 M_{\odot}$ is incorrect; for $1 M_{\odot}$ stars, observed ${ }^{12} \mathrm{C} /{ }^{13} \mathrm{C}$ ratios are a factor of $\sim 3$ below calculated values (Fig. 1). A wealth of papers have interpreted this as implying some form of extra mixing to bring up more ${ }^{13} \mathrm{C}$ (recently, see, e.g., Charbonnel 1994; Wallerstein \& Morell 1994; and references therein); observations (Gilroy \& Brown 1991) show that this must take place subsequent to first dredge-up, since low ${ }^{12} \mathrm{C} /{ }^{13} \mathrm{C}$ values do not appear until then.

For ${ }^{17} \mathrm{O} /{ }^{16} \mathrm{O}$ and ${ }^{18} \mathrm{O} /{ }^{16} \mathrm{O}$, first and second dredge-up theory is not in conflict with observations (Dearborn, Tinsley, \& Schramm 1978; Landré et al. 1990; Dearborn 1992; Schaller et al. 1992; Bressan et al. 1993; El Eid 1994; Boothroyd, Sackmann, \& Wasserburg 1994, hereafter BSW-I). However, a discrepancy does exist in the later asymptotic giant branch (AGB) phase. It has been shown that there is an "inaccessible" region in the ${ }^{17} \mathrm{O} /{ }^{16} \mathrm{O}$ versus ${ }^{18} \mathrm{O} /{ }^{16} \mathrm{O}$ diagram (see Fig. 2) which cannot be populated by standard dredge-up or hot bottom burning (Boothroyd, Sackmann, \& Wasserburg 1995, hereaf- ter BSW-II). However, observations show that AGB stars lie in this region (Harris, Lambert, \& Smith 1985; Harris et al. 1987; Kahane et al. 1992). The problem is intensified by precise O-isotope data of circumstellar $\mathrm{Al}_{2} \mathrm{O}_{3}$ grains, preserved in meteorites, that are believed to come from AGB stars (Huss et al. 1992, 1994; Nittler et al. 1994; L. R. Nittler, private communication); some data lie in the "inaccessible" region (see Fig. 2). BSW-II pointed out that this puzzle might also be solved by some form of extra mixing ("cool bottom processing"), in which the bottom of the convective envelope remains cool while an ad hoc mixing mechanism brings material down to layers hot enough for some nuclear processing. The data require that this take place in stars of $1-2 M_{\odot}$. There is a large body of literature on possible mixing mechanisms, particularly for $\mathrm{C}$ and $\mathrm{Li}$ (see, e.g., Dearborn et al. 1976; Genova \& Schatzmann 1979; Press \& Rybicki 1981; Pinsonneault et al. 1989; Zahn 1992).

\section{METHODS AND RESULTS}

Parametric computations were performed, with envelope structures obtained from models of a $1 M_{\odot}$ star (Sackmann, Boothroyd, \& Kraemer 1993), not long after first dredge-up on the RGB, and prior to the first He-shell flash (thermal pulse) on the AGB. Table 1 shows the initial stellar mass $M_{i}$, luminosity $L$, degenerate core mass $M_{c}$, rate $\dot{M}_{c}$ at which the $\mathrm{H}$-shell burns outward in mass, temperature $\log T_{H}$ at the base of the H-shell, and time $\tau^{*}=\tau_{\mathrm{RGB}}^{*}$ or $\tau_{\mathrm{AGB}}^{*}$ spent on the RGB 


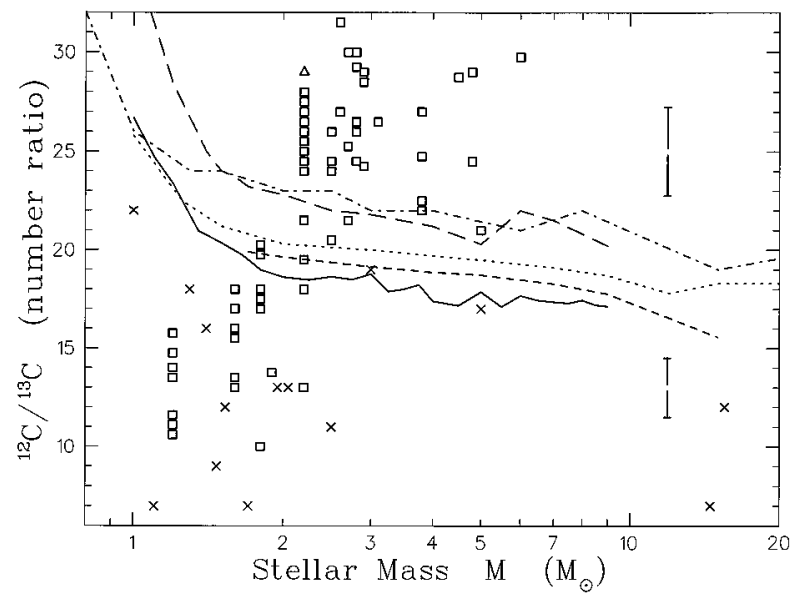

FIG. 1.-Comparison between observations and first dredge-up theory for ${ }^{12} \mathrm{C} /{ }^{13} \mathrm{C}$ in red giants as a function of stellar mass $M$. Isolated stars (crosses) of Harris \& Lambert (1984a, b) and Harris, Lambert, \& Smith (1988) have much less accurate stellar masses than open cluster data (open squares; lower limit: open triangle) of Gilroy (1989); typical ${ }^{12} \mathrm{C} /{ }^{13} \mathrm{C}$ uncertainties are shown by error bars at right. Theory as follows: solid line, Boothroyd \& Sackmann (1995); short dashed line, El Eid (1994); long dashed line, Bressan et al. (1993); dotted line, Schaller et al. (1992); dot-dashed line, Dearborn (1992).

or AGB, respectively. Initial RGB and AGB envelope compositions were obtained from full stellar evolutionary calculations of Boothroyd \& Sackmann (1995). Nuclear rates of Caughlan \& Fowler (1988) were used, except for ${ }^{17} \mathrm{O}+p$, where those of Landré et al. (1990) were used.

In our two-stream "conveyor-belt" circulation model, matter from the bottom of envelope convection streamed downward, reaching a maximum temperature $T_{P}$, then returned upward and was mixed with the convective envelope (i.e., a composition advection equation with nuclear burning, and no mixing between downward and upward streams). Envelope and stream compositions were followed through time. The value of $T_{P}$ was treated as a free parameter (see Table 1); values selected for discussion were those satisfying the observational data. The other key free parameter was the stream mass flow rate $\dot{M}_{P}$. This must be slower than that of RGB or AGB convective envelopes $\left(\dot{M}_{P} \ll \dot{M}_{\text {conv }} \sim 1 M_{\odot} \mathrm{yr}^{-1}\right)$, while the streams must move faster than the speed with which the H-shell burns its way outward $\left(\dot{M}_{P} \gtrsim \dot{M}_{c}\right.$ : see Table 1$)$. We explored a wide range of $\dot{M}_{P}$ values (see Table 1 ). We also explored the effect of varying the envelope mass $M_{E}$ on the AGB, where $M_{E}$ is uncertain as a result of the stellar wind mass-loss rate.

For equal downward and upward velocities, the fractional areas at the base of the convective envelope occupied by downward and upward streams are equal. The downward stream then spends a time $\Delta t_{d}=0.5 \Delta M_{r} / \dot{M}_{P}$ in a layer $\Delta M_{r}$, the upward stream spending the same time $\Delta t_{u}$ there on its way out. When downward and upward velocities and respective fractional areas $f_{d}$ and $f_{u}$ are not equal, these times become $\Delta t_{d}=f_{d} \Delta M_{r} / \dot{M}_{P}$ and $\Delta t_{u}=f_{u} \Delta M_{r} / \dot{M}_{P}$. The total time spent in any mass layer is independent of $f_{d}$ and $f_{u}$, for $f_{d}+f_{u}=1$; note that $f_{d}+f_{u}<1$ is not a separate case, being equivalent to increasing $\dot{M}_{P}$ while reducing the timescale $\tau^{*}$. Besides $f_{d}=f_{u}=0.5$, we considered $f_{u} / f_{d}=9$ and $f_{u} / f_{d}=99$. Even such large variations in $f_{u} / f_{d}$ made essentially no difference to the resulting envelope abundances, except for ${ }^{7} \mathrm{Li}$.

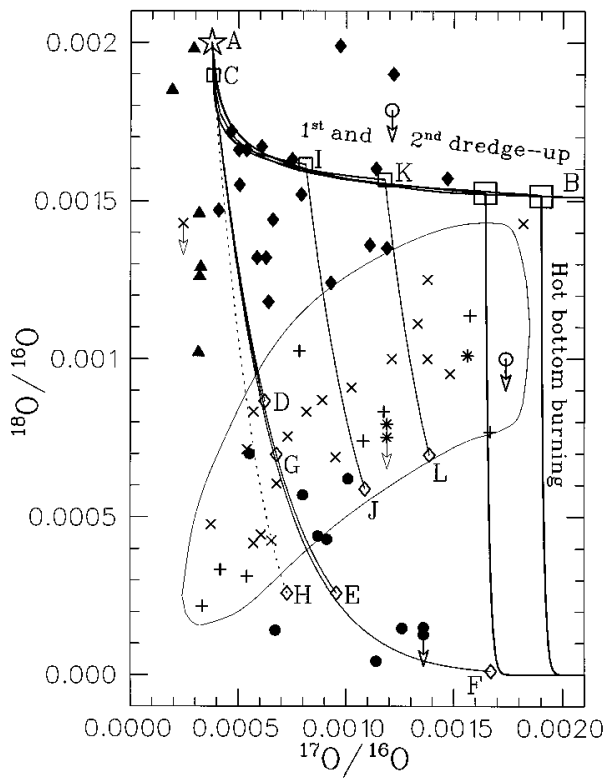

FIG. 2.-Comparison of predicted ${ }^{18} \mathrm{O} /{ }^{16} \mathrm{O}$ and ${ }^{17} \mathrm{O} /{ }^{16} \mathrm{O}$ with grain and stellar observations. Two J-type carbon stars (circles), with ${ }^{12} \mathrm{C} /{ }^{13} \mathrm{C} \sim 3$ indicating hot bottom burning, have only upper limits for ${ }^{18} \mathrm{O}$. Normal S stars (plus signs) and C stars (crosses) on the AGB have large errors $\sim 50 \%$ (not shown); circumstellar C star observations (asterisks) of Kahane et al. (1992) are somewhat more accurate. The loop shows where most $\mathrm{C}$ and $\mathrm{S}$ star observations lie. High-precision grain measurements (uncertainties $\sim 10 \%$ ) are shown by filled symbols, grouped as by Nittler et al. (1994): group 1 (diamonds) are consistent with first and second dredge-up (given some metallicity variation, which shifts point A: see BSW-I and BSW-II), group 2 ( filled circles) display very low ${ }^{18} \mathrm{O} /{ }^{16} \mathrm{O}$ (requiring ${ }^{18} \mathrm{O}$ destruction), and group 3 (triangles at left) display low ${ }^{17} \mathrm{O} /{ }^{16} \mathrm{O}$. Initial isotope ratios are for the large star at $\mathrm{A}$; first and second dredge-up shift compositions along the line AB. Termination of first and second dredge-up for 6 and $7 M_{\odot}$ stars are shown by large open squares; attached vertical lines show the effect of hot bottom burning on the AGB (BSW-II). Note that the region to their left, where many observations lie, cannot be populated via standard dredge-up and hot bottom burning. "Cool bottom processing" can populate this otherwise inaccessible region: termination of first dredge-up for $1,1.5$, and $1.65 M_{\odot}$ stars is shown by small open squares at $\mathrm{C}, \mathrm{I}$, and $\mathrm{K}$, respectively, and the effects of cool bottom processing are shown by curves slanting down and to the right. For $1 M_{\odot}$ : CD, log $T_{P}=7.49 ; \mathrm{CE}, \log T_{P}=7.51 ; \mathrm{CF}, \log T_{P}=7.53$, all with $M_{E}=0.2 M_{\odot} ; \mathrm{CG}$, same as $\mathrm{CE}$; with $M_{E}=0.4 M_{\odot}$; dotted curve $\mathrm{CH}$, same as $\mathrm{CE}$, but with highest allowed ${ }^{17} \mathrm{O}$-destruction rate. Curve IJ: $1.5 M_{\odot}$ with $M_{E}=0.4 M_{\odot} ; \mathrm{KL}, 1.65 M_{\odot}$ with $M_{E}=0.5 M_{\odot}$, both with $\log T_{P}=7.51$. Endpoints (diamonds) are for $\tau_{\mathrm{AGB}}^{*}=10^{6} \mathrm{yr}$.

Slow deep mixing tends to be opposed by a gradient in the mean molecular weight $\mu$. A $\mu$-discontinuity is left behind by first dredge-up; only after the H-burning shell reaches and destroys this "barrier" can circulation reach the higher temperature domains (see, e.g., Charbonnel 1994). We infer that our reported $T_{P}$ values are determined by the $\mu$-gradient in the outer wing of the $\mathrm{H}$-shell that inhibits mixing. For stellar masses above $\sim 2.3 M_{\odot}$, the RGB ends before the $\mathrm{H}$-shell reaches the $\mu$-discontinuity-no RGB cool bottom processing should take place in such stars. On the early AGB, not too much cool bottom processing is expected, since the $\mathrm{H}$-shell largely stops burning; one does expect cool bottom processing later on the AGB, when the H-shell burns strongly again (intermittently interrupted by He-shell flashes).

The calculated envelope composition depended sensitively on $T_{P}$. In the relevant RGB regime of partial CNO processing, a change of $\Delta \log T_{P}=0.03$ resulted in a factor of 2 change in 
TABLE 1

RGB AND AGB ENVElope Parameters

\begin{tabular}{|c|c|c|c|c|c|c|c|c|c|c|}
\hline Branch & $\begin{array}{c}M_{i} \\
\left(M_{\odot}\right)\end{array}$ & $\log L$ & $\begin{array}{c}M_{c} \\
\left(M_{\odot}\right)\end{array}$ & $\begin{array}{c}\dot{M}_{c} \\
\left(M_{\odot} \mathrm{yr}^{-1}\right)\end{array}$ & $\log T_{H}$ & $\begin{array}{c}\tau^{*} \\
(\mathrm{yr})\end{array}$ & $\begin{array}{c}M_{E} \\
\left(M_{\odot}\right)\end{array}$ & $\log T_{P}$ & $\begin{array}{c}\dot{M}_{P} \\
\left(M_{\odot} \mathrm{yr}^{-1}\right)\end{array}$ & $f_{u} / f_{d}$ \\
\hline RGB & 1.0 & 1.6 & 0.251 & $6 \times 10^{-10}$ & 7.473 & $5 \times 10^{7}$ & 0.7 & $7.24-7.36$ & $10^{-9}-10^{-4}$ & $1,9,99$ \\
\hline \multirow{3}{*}{ AGB $\ldots \ldots \ldots \ldots \ldots$} & 1.0 & 3.2 & 0.536 & $3 \times 10^{-8}$ & 7.679 & $10^{6}$ & $0.2,0.4$ & $7.44-7.60$ & $10^{-7}-10^{-4}$ & $1,9,99$ \\
\hline & 1.5 & $\ldots$ & $\ldots$ & $\ldots$ & $\ldots$ & $\ldots$ & $0.4,0.8$ & $7.51,7.53$ & $10^{-5} \cdot 10^{-4}$ & 1 \\
\hline & 1.65 & $\ldots$ & $\ldots$ & $\ldots$ & $\ldots$ & $\ldots$ & $0.5,1.0$ & $7.51,7.53$ & $10^{-5}, 10^{-4}$ & 1 \\
\hline
\end{tabular}

${ }^{12} \mathrm{C} /{ }^{13} \mathrm{C}$. Consistency between theory and observations was achieved for $\log T_{P} \approx 7.30$. For $\dot{M}_{P} \gtrsim 10^{-8} M_{\odot} \mathrm{yr}^{-1},{ }^{13} \mathrm{C} /{ }^{12} \mathrm{C}$ and ${ }^{12} \mathrm{C}$ were essentially independent of $\dot{M}_{P}$ (and of $f_{u} / f_{d}$ ) for our RGB model. Results of our mass transport scheme are thus resilient to details of the motion except for $T_{P}$ and a minimum mass transfer rate. Full dynamical models for deep mixing should thus yield results similar to the two-stream model for similar $T_{P}$. The $\dot{M}_{P}$ independence is due to the low extent of nuclear processing on each circulation pass for these speeds of transport. An isotope of abundance $X$ that burns at a rate $R_{X, r}$ will be reduced by $\Delta X_{r, d}=-X R_{X, r} \Delta t_{d}$ as it moves down through a layer $\Delta M_{r}$ (and similarly by $\Delta X_{r, u}$ on the way back up). If the total change $\Delta X$ per pass is small, then $\Delta X=\Sigma_{r}\left(\Delta X_{r, d}+\Delta X_{r, u}\right) \approx-\left(X / \dot{M}_{P}\right) \Sigma_{r}\left[\left(f_{d}+f_{u}\right) R_{X, r} \Delta M_{r}\right] \equiv$ $-X \lambda_{X} / \dot{M}_{P}$, where $\lambda_{X}$ is independent of $M_{P}$, being the burning rate integrated over mass (not time!) through the path. If $X$ is also produced by burning isotope $Y$, one obtains $\Delta X=\left(Y \lambda_{Y}-X \lambda_{X}\right) / \dot{M}_{P}$. Matter is delivered to the envelope at a rate $\dot{M}_{P}$, where it is diluted by the envelope mass $M_{E}$. Thus, in the envelope, $d X_{E} / d t=\left(\dot{M}_{P} / M_{E}\right) \Delta X=\left(Y \lambda_{Y}-X \lambda_{X}\right) / M_{E}$, independent of $\dot{M}_{P}$. This implies $d^{12} \mathrm{C}_{E} / d t=-\lambda_{12}{ }^{12} \mathrm{C}_{E} / M_{E}$ and $d^{13} \mathrm{C}_{E} / d t=\left(\lambda_{12}{ }^{12} \mathrm{C}_{E}-\lambda_{13}{ }^{13} \mathrm{C}_{E}\right) / M_{E}$, in the approximation of small burning per pass. Solving for envelope ${ }^{12} \mathrm{C}$ and ${ }^{13} \mathrm{C}$ abundances yields

$$
\begin{aligned}
{ }^{13} \mathrm{C}_{E}(t) & { }^{12} \mathrm{C}_{E}(t)={ }^{12} \mathrm{C}_{E}(0) e^{-\lambda_{12} t / M_{E}}, \\
& \frac{{ }^{12} \mathrm{C}_{E}(0)}{{ }^{12}(0)} e^{-\left(\lambda_{13}-\lambda_{12}\right) t / M_{E}} \\
& +\frac{\lambda_{12}}{\lambda_{13}-\lambda_{12}}\left(1-e^{-\left(\lambda_{13}-\lambda_{12}\right) t / M_{E}}\right) .
\end{aligned}
$$

Equation (2) can be further simplified: ${ }^{13} \mathrm{C}$ burns $\sim 3.5$ times as fast as ${ }^{12} \mathrm{C}$ (almost independent of temperature), so $\lambda_{13} /$ $\lambda_{12} \approx 3.5$, and $\lambda_{12} /\left(\lambda_{13}-\lambda_{12}\right) \approx 0.4$. For $\dot{M}_{P} \gg M_{E} / \tau_{\mathrm{RGB}}^{*}$, the above equations are in excellent agreement with full numerical calculations.

Figure 3 illustrates our RGB model results for $\log$ $T_{P} \approx 7.30$. Envelope ${ }^{12} \mathrm{C} /{ }^{13} \mathrm{C}$ drops exponentially from 27 (as left by first dredge-up) to $\sim 5$, spending much time in the range $15 \gtrsim{ }^{12} \mathrm{C} /{ }^{13} \mathrm{C} \gtrsim 7$ where most observations for $\sim 1 M_{\odot}$ stars lie (see Fig. 1). From 1 to $\sim 2.3 M_{\odot}$, increasing the stellar mass implies larger $M_{E}$ and shorter $\tau_{\mathrm{RGB}}^{*}$, and thus higher ${ }^{12} \mathrm{C} /{ }^{13} \mathrm{C}$, in agreement with the observed trend (recall that no cool bottom processing is expected above $\sim 2.3 M_{\odot}$ ). We conclude that excellent agreement is obtained between the observations and this cool bottom processing model, in which $\log T_{P}$ differs by $\Delta \log T \approx 0.17$ from the $\mathrm{H}$-shell temperature $\log T_{H}$ (see Table 1). Note that ${ }^{12} \mathrm{C}$ and ${ }^{14} \mathrm{~N}$ abundances change by only $\sim 20 \%$, while $\mathrm{O}$ isotopes are essentially unaffected. The ${ }^{9} \mathrm{Be}$ abundance is decreased; ${ }^{7} \mathrm{Li}$ may either decrease or increase via the Cameron-Fowler mechanism, depending on $\dot{M}_{P}$ and $f_{u} / f_{d}$ (a future paper will discuss this).
Big bang nucleosynthesis models convert an upper bound on primordial $\left(\mathrm{D}+{ }^{3} \mathrm{He}\right) / \mathrm{H}$ into a lower bound on the baryon density $\Omega_{b}$. To infer primordial (D $\left.+{ }^{3} \mathrm{He}\right) / \mathrm{H}$, one must consider galactic chemical evolution. Deuterium is burned to ${ }^{3} \mathrm{He}$ during protostellar collapse, but this does not affect the sum $\left(\mathrm{D}+{ }^{3} \mathrm{He}\right)$ on the main sequence. However, first dredge-up substantially enhances ${ }^{3} \mathrm{He}$, by a factor of $\sim 6$ for $1 M_{\odot}$, and by $\sim 2$ for $2 M_{\odot}$; only above $\sim 4 M_{\odot}$ is ${ }^{3} \mathrm{He}$ depleted (Iben 1967; Boothroyd \& Sackmann 1995). As low-mass stars are more common, stellar processing would increase galactic (D + $\left.{ }^{3} \mathrm{He}\right) / \mathrm{H}$; the presolar value has thus been used as an upper limit to the primordial value. Hogan (1995) pointed out that $\mathrm{CNO}$ processing sufficient to explain the ${ }^{12} \mathrm{C} /{ }^{13} \mathrm{C}$ observations in low-mass stars should also destroy ${ }^{3} \mathrm{He}$, perhaps resulting in net reduction of $\left(\mathrm{D}+{ }^{3} \mathrm{He}\right) / \mathrm{H}$. Our models confirm this. For a $1 M_{\odot}$ star, after first dredge-up ${ }^{3} \mathrm{He}$ enhancement (by $\sim 6$ ), RGB cool bottom processing reduces ${ }^{3} \mathrm{He}$ by a factor of $\sim 10$, yielding net depletion by a factor of $\sim 2$. Both enhancement and reduction are less for higher stellar masses, but probably still result in $\left(\mathrm{D}+{ }^{3} \mathrm{He}\right)$ depletion for stars of $\lesssim 2 M_{\odot}$. Hence, stellar processing should reduce galactic $\left(\mathrm{D}+{ }^{3} \mathrm{He}\right) / \mathrm{H}$. The primordial value must thus be larger than the present estimate, removing the strongest lower bound on the baryon density.

For the AGB, envelope compositions were also nearly independent of $\dot{M}_{P}$ and $f_{d} / f_{u}$ but depended sensitively on $T_{P}$ (see points D, E, and F in Fig. 2). A temperature of $\log$ $T_{P} \approx 7.51$ was able to populate the hitherto inaccessible region of the ${ }^{18} \mathrm{O} /{ }^{16} \mathrm{O}$ versus ${ }^{17} \mathrm{O} /{ }^{16} \mathrm{O}$ diagram (curves $\mathrm{CE}, \mathrm{IJ}$, and $\mathrm{KL}$ in Fig. 2), achieving excellent agreement with observed $\mathrm{O}$ isotope ratios. Note that this $T_{P}$ differs by the same amount $\Delta \log T=0.17$ from the AGB H-shell temperature as on the $\mathrm{RGB}$, as expected from the $\mu$-gradient argument. Figure 4 shows the results: ${ }^{18} \mathrm{O} /{ }^{16} \mathrm{O}$ drops by a factor of $\sim 7$, and ${ }^{17} \mathrm{O} /{ }^{16} \mathrm{O}$ grows by a factor of $\sim 2.5$ (see curve $\mathrm{CE}$ of Fig. 2). The ${ }^{18} \mathrm{O}$ abundance decays exponentially with time on the AGB (as does ${ }^{12} \mathrm{C}$ on the RGB). The behavior of ${ }^{17} \mathrm{O}$ is governed by an equation similar to that of ${ }^{13} \mathrm{C}$ on the RGB.

The effect of AGB cool bottom processing on $\mathrm{C}$ isotopes is of key interest. This is complicated by periodic He-shell flashes, which can dredge up significant amounts of ${ }^{12} \mathrm{C}$ ("third dredge-up") and may produce a carbon star. For a $1 M_{\odot}$ star, the core mass increases by $\sim 0.003 M_{\odot}$ as a result of H-shell burning in each $\sim 10^{5} \mathrm{yr}$ period between flashes (Sackmann et al. 1993). Typically, one might expect $\sim \frac{1}{3}$ of this (of which $25 \%$ by mass is ${ }^{12} \mathrm{C}$ ) to be dredged up in each flash. Such dredge-up was added parametrically to our models, as shown by the sawtoothed curves of $\mathrm{C} / \mathrm{O}$ (labeled " $\mathrm{dr}$ ") and ${ }^{12} \mathrm{C} /{ }^{13} \mathrm{C}$ (labeled "dr") of Figure 4; the smooth curves (labeled "no") are for no third dredge-up. Reasonable amounts of third dredge-up may still yield a carbon star, despite ${ }^{12} \mathrm{C}$ destruction; the case of Figure 4 is an S star for the first half of its AGB lifetime, and a C star for the second half. However, when cool bottom processing occurs, obtaining $\mathrm{C} / \mathrm{O}>1$ may require more car- 


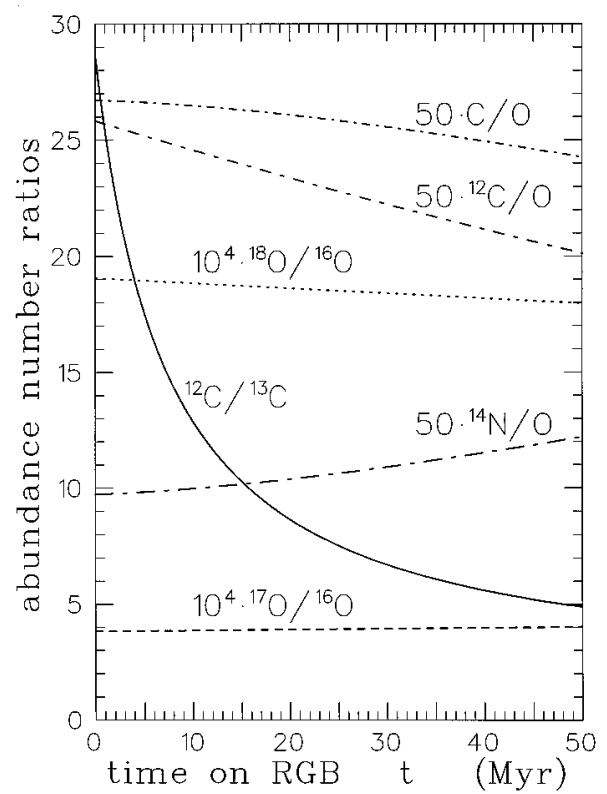

FIG. 3.-Envelope composition as a function of time on the RGB for a $1 M_{\odot}$ star with envelope mass $M_{E}=0.7 M_{\odot}$, for mixing reaching a temperature $\log T_{P}=7.30$ with mass flow rate $M_{P}=10^{-4} M_{\odot} \mathrm{yr}^{-1}$, and $f_{d}=f_{u}=0.5$.

bon dredge-up per flash than is generally assumed (see Busso et al. 1993), making it more difficult to produce carbon stars of $\sim 1 M_{\odot}$. In addition, AGB cool bottom processing results in ${ }^{12} \mathrm{C} /{ }^{13} \mathrm{C} \sim 4$, even when third dredge-up is present. This large envelope ${ }^{13} \mathrm{C}$ does not help with the puzzle of the $s$-process ${ }^{13} \mathrm{C}$ neutron source, since the $\mathrm{H}$-shell burns ${ }^{13} \mathrm{C}$ to ${ }^{14} \mathrm{~N}$. Indeed, it poses a new puzzle, namely, how to account for AGB stars with low ${ }^{18} \mathrm{O} /{ }^{16} \mathrm{O}$ but high ${ }^{12} \mathrm{C} /{ }^{13} \mathrm{C}$. Note that cool bottom processing also results in large ${ }^{14} \mathrm{~N}$ enrichments, by a factor of 3-6.

Full stellar evolutionary models that include some dynamical prescription for extra mixing are needed to investigate the interaction between cool bottom processing, third dredge-up, and $\mathrm{C}$ star formation. However, such models should yield results similar to our parametric models, if the mixing reaches a similar temperature $T_{P}$; the increase in $T_{P}$ as stars climb the RGB or AGB should be largely offset by the faster timescales $\tau^{*}$, since different CNO-burning rates have similar temperature dependences.

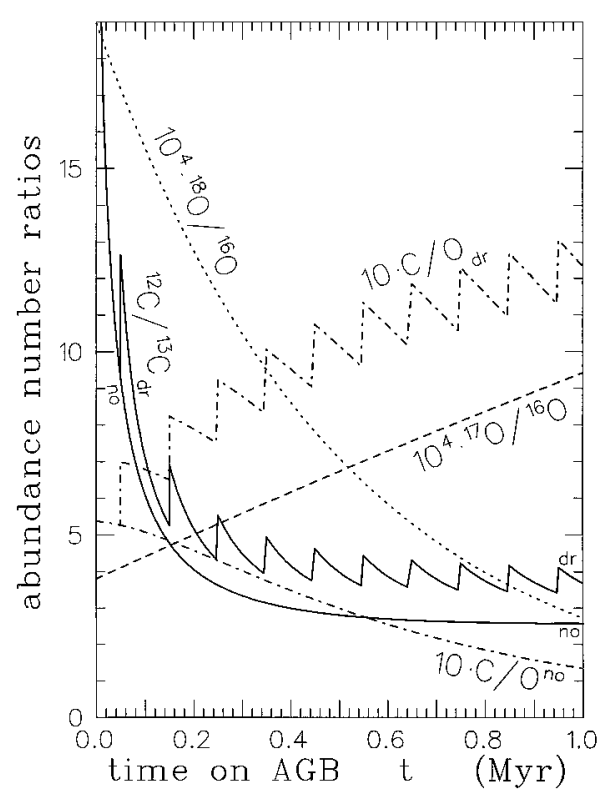

FIG. 4.-Envelope composition as a function of time on the AGB for a $1 M_{\odot}$ star with $M_{E}=0.2 M_{\odot}, \log T_{P}=7.51, \dot{M}_{P}=10^{-4} M_{\odot} \mathrm{yr}^{-1}$, and $f_{d}=f_{u}=0.5$. (Note that starting from the final RGB cool bottom processing composition, rather than the first dredge-up composition, would be equivalent to chopping off the left-hand $10 \%$ of the graph). Note the regular decrease in ${ }^{18} \mathrm{O} /{ }^{16} \mathrm{O}$ and increase in ${ }^{17} \mathrm{O} /{ }^{16} \mathrm{O}$; these are unaffected by third dredge-up. The curves labeled "no" correspond to evolution without third dredge-up and show a decrease in $\mathrm{C} / \mathrm{O}$ and ${ }^{12} \mathrm{C} /{ }^{13} \mathrm{C}$. Sawtoothed curves labeled "dr" include periodic third dredge-up, which yields increasing $\mathrm{C} / \mathrm{O}$ while leaving low ${ }^{12} \mathrm{C} /{ }^{13} \mathrm{C}$.

This paper was much improved by penetrating comments and creative suggestions by M. Busso and critical reading and comments by G. Huss, G. Bazan, C. A. Barnes, R. F. Christy, and R. Gallino. A. I. B. wishes to thank S. D. Tremaine and P. G. Martin for the support provided by the Canadian Institute for Theoretical Astrophysics. This work was supported in part by a grant from the Natural Sciences and Engineering Research Council of Canada, NASA grants NAGW-3040, NAGW-3337, NAGW-3297, and NSF grant PHY 94-20470. Contribution No. 5498(892), Division of Geological and Planetary Sciences of Caltech.

\section{REFERENCES}

Boothroyd, A. I., \& Sackmann, I.-J. 1995, in preparation Boothroyd, A. I., Sackmann, I.-J., \& Wasserburg, G. J. 1994, ApJ, 430, L77 (BSW-I)

1995, ApJ, 442, L21 (BSW-II)

Bressan, A., Fagotto, F., Bertelli, G., \& Chiosi, C. 1993, A\&AS, 100, 647

Busso, M., Chieffi, A., Gallino, R., Limougi, M., Raiteri, C. M., \& Straniero, O. 1993, in IAU Symposium 153: Planetary Nebulae, ed. R. Weinberg \& A Acker (Dordrecht: Kluwer), 361

Caughlan, G. R., \& Fowler, W. A. 1988, Atomic Data Nucl. Data Tables, 40, 205

Charbonnel, C. 1994, A\&A, 282, 811

Dearborn, D., Eggleton, P. P., \& Schramm, D. N. 1976, ApJ, 203, 455

Dearborn, D., Tinsley, B. M., \& Schramm, D. N. 1978, ApJ, 223, 557

Dearborn, D. S. P. 1992, Phys. Rep., 210, 367

El Eid, M. F. 1994, A\&A, 285, 915

Genova, F., \& Schatzmann, E. 1979, A\&A, 78, 323

Gilroy, K. K. 1989, ApJ, 347, 835

Gilroy, K. K., \& Brown, J. A. 1991, ApJ, 371, 578

Harris, M. J., \& Lambert, D. L. 1984a, ApJ, 281, 739

$\longrightarrow$. 1984b, ApJ, 285, 674
Harris, M. J., Lambert, D. L., Hinkle, K. H., Gustafsson, B., \& Eriksson, K. 1987, ApJ, 316, 294

Harris, M. J., Lambert, D. L., \& Smith, V. V. 1985, ApJ, 299, 375

. 1988, ApJ, 325, 768

Hogan, C. J. 1995, ApJ, 441, L17

Huss, G. R., Fahey, A. J., Gallino, R., \& Wasserburg, G. J., 1994, ApJ, 430, L81

Huss, G. R., Hutcheon, I. D., Wasserburg, G. J., \& Stone, J. 1992, Lunar Planet. Sci., 23, 563

Iben, I., Jr. 1967, ApJ, 147, 624

Kahane, C., Cernicharo, J., Gómez-González, J., \& Guélin, M. 1992, A\&A, 256, 235

Landré, V., Prantzos, N., Aguer, P., Bogaert, G., Lefebvre, A., \& Thibaud, J. P. 1990, A\&A, 240, 85

Nittler, L. R., Alexander, C. M. O'D., Gao, X., Walker, R. M., \& Zinner, E. K. 1994, Nature, 370, 443

Pinsonneault, M. H., Kawaler, S. D., Sofia, S., \& Demarque, P. 1989, ApJ, 338, 424 Press, W. H., \& Rybicki, G. B. 1981, ApJ, 248, 751

Sackmann, I.-J., Boothroyd, A. I., \& Kraemer, K. E. 1993, ApJ, 418, 457

Schaller, G., Schaerer, D., Meynet, G., \& Maeder, A. 1992, A\&AS, 96, 269

Wallerstein, G., \& Morell, O. 1994, A\&A, 281, L37

Zahn, J. P. 1992, A\&A, 265, 115 